Research Paper

\title{
Inhibition of Autophagy Signaling via 3-methyladenine Rescued Nicotine-Mediated Cardiac Pathological Effects and Heart Dysfunctions
}

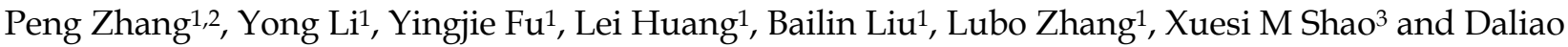 \\ $\mathrm{Xiao}^{1 凶}$ \\ 1. Lawrence D. Longo, MD Center for Perinatal Biology, Department of Basic Sciences, Loma Linda University School of Medicine, Loma Linda, California, \\ USA \\ 2. Department of Cardiology, The First Affiliated Hospital of Chongqing Medical University, Chongqing, China \\ 3. Department of Neurobiology, David Geffen School of Medicine at UCLA, University of California at Los Angeles, Los Angeles, California, USA \\ $\triangle$ Corresponding author: Daliao Xiao, PhD, Lawrence D. Longo, MD Center for Perinatal Biology, Department of Basic Sciences, Loma Linda University School \\ of Medicine, Loma Linda, CA 92350. Tel: 909-558-4325; Fax: 909-558-4029; E-mail: Dxiao@llu.edu
}

(C) The author(s). This is an open access article distributed under the terms of the Creative Commons Attribution License (https://creativecommons.org/licenses/by/4.0/). See http://ivyspring.com/terms for full terms and conditions.

Received: 2019.10.17; Accepted: 2020.01.31; Published: 2020.02.21

\begin{abstract}
Rationale: Cigarette smoking is a well-established risk factor for myocardial infarction and sudden cardiac death. The deleterious effects are mainly due to nicotine, but the mechanisms involved and theranostics remain unclear. Thus, we tested the hypothesis that nicotine exposure increases the heart sensitivity to ischemia/reperfusion injury and dysfunction, which can be rescued by autophagy inhibitor.

Methods: Nicotine or saline was administered to adult rats via subcutaneous osmotic minipumps in the absence or presence of an autophagy inhibitor, 3-methyladenine (3-MA). After 30 days of nicotine treatment, the rats underwent the cardiac ischemia/reperfusion (I/R) procedure and echocardiography analysis, and the heart tissues were isolated for molecular biological studies.

Results: Nicotine exposure increased I/R-induced cardiac injury and cardiac dysfunction as compared to the control. The levels of autophagy-related proteins including LC3 II, P62, Beclin1, and Atg5 were upregulated in the reperfused hearts isolated from nicotine-treated group. In addition, nicotine enhanced cardiac and plasma ROS production, and increased the phosphorylation of GSK3 $\beta$ (ser9) in the left ventricle tissues. Treatment with 3-MA abolished nicotine-mediated increase in the levels of autophagy-related proteins and phosphorylation of GSK3 $\beta$, but had no effect on ROS production. Of importance, 3-MA ameliorated the augmented I/R-induced cardiac injury and dysfunction in the nicotine-treated group as compared to the control.

Conclusion: Our results demonstrate that nicotine exposure enhances autophagy signaling pathway, resulting in development of ischemic-sensitive phenotype of heart. It suggests a potentially novel therapeutic strategy of autophagy inhibition for the treatment of ischemic heart disease.
\end{abstract}

Key words: nicotine, cardiac ischemia/reperfusion injury, autophagy pathway

\section{Introduction}

Nicotine exposure, either from tobacco cigarette smoking or nicotine use (nicotine gum or patch) has become one of the most pressing public concerns in modern life. Furthermore, electronic cigarette (e-cigarette), an electronic nicotine delivery system has been introduced in the global market during the past ten years. Thus, nicotine addiction and nicotine abuse are becoming a potential public health concern in the $21^{\text {st }}$ century. Epidemiologic studies have shown that cigarette smoking is the most prominent risk factor for coronary heart disease and cardiovascular mortality in the general population [1-4]. Although cigarette smoke contains thousands of chemicals, nicotine and its metabolites are recognized as being 
the major psychoactive compounds and are likely to contribute to the development of cardiovascular disorders. Indeed, clinical and animal studies have suggested that cigarette smoking-induced myocardial ischemia is mediated by nicotine [5-7]. However, underlying mechanisms of cardiac dysfunction induced by nicotine exposure are largely unknown and the specific pathophysiologic pathways leading to the development of heart ischemic-sensitive phenotype remain to be identified.

Autophagy is a cellular process associated with unnecessary protein damage and organelle degradation. Autophagy functions as a protein quality controller and cellular homeostasis keeper [8]. In the heart, autophagy occurs constitutively at a basal level [9], but is enhanced during pathological conditions, including cardiac hypertrophy, cardiomyopathy, ischemic heart disease and heart failure [10]. However, the role of autophagy in ischemic heart injury is controversial and complex [11]. Whether autophagy is protective or detrimental is context-dependent. In general, a modest increase in autophagy under ischemic stress appears to be protective, whereas massive activation may be detrimental and induce type 2 cell death (autophagy-induced cell death), which contributes to ischemic cardiac injury [12]. Previous studies have shown that cigarette smoke and nicotine exposure cause different tissue and cell damage via activation of autophagy pathway [13-16]. This led us to contemplate if chronic nicotine exposure alters the homeostatic autophagy process and exert adverse effects in cardiomyocytes.

Therefore, in the present study we first evaluated whether chronic nicotine exposure increased the heart susceptibility to ischemia/reperfusion (I/R) injury and decreased heart functional recovery after $I / R$ injury. Next, we examined whether chronic nicotine exposure enhanced autophagy-related biomarkers in cardiomyocytes. Finally, we assessed the effect of therapeutic inhibition of autophagy activation on nicotine-mediated cardiac ischemic injury and dysfunction to test the hypothesis that nicotinemediated autophagy plays a causal role in the development of heart ischemic-sensitive phenotype in an adult rat model. Our findings suggest that autophagy may be a potential therapeutic molecular target for the treatment of cardiovascular ischemic disease.

\section{Methods}

\section{Experimental animals}

The procedures and protocols were approved by the Institutional Animal Care and Use Committee of Loma Linda University. All animal studies followed the guidelines in the National Institutes of Health Guide for the Care and Use of Laboratory Animals. Sprague-Dawley male rats (8-months-old) were purchased from Charles River Laboratories (Portage, MI) and housed individually in Plexiglas acrylic cages, located in air-conditioned rooms (room temperature $22^{\circ} \mathrm{C}$, relative humidity $60 \%$; lights on from 8:00 a.m. to 8:00 p.m.). Pellet food and tap water were available ad libitum. Rats were anesthetized with isoflurane (5\% for induction, $2 \%$ for maintenance) and adequate anesthesia was determined by the loss of pedal withdrawal reflex and other reactions from the animals in response to pinching the toe or tail. Saline or nicotine (at $4 \mu \mathrm{g} / \mathrm{kg} / \mathrm{min}$ ) were individually administered to rats through osmotic minipumps (type 2ML4, Alzet, Durect Corp., Cupertino, CA) and inserted under the skin on the back of rats for 30 days, as previously described in detail [17-19]. The dose of nicotine resulted in blood levels closely resembling those occurring in moderate human smokers [20]. A total of 45 rats were used in this project and were randomly divided into four groups: 1) saline control $(\mathrm{n}=13) ; 2)$ nicotine $(\mathrm{n}=10)$; 3) saline + 3-MA $(\mathrm{n}=11)$; and 4) nicotine + 3-MA ( $\mathrm{n}=11)$. One week before I/ $\mathrm{R}$ surgery, 3-methyladenine (3-MA) (15 mg/kg/day; Sigma Aldrich, St. Louis, MO) or saline was respectively administered to rats via intraperitoneal (i.p.) injection for seven days. Previous studies have shown that treatment with this dosage of 3-MA significantly inhibits autophagy signaling [21, 22]. To separate the confound effect of sex, in this study we only selected male rats in the proposed experiments. In our future studies, we will investigate the effect of nicotine on female rats.

\section{Echocardiography}

To measure LV function and heart chamber dimensions in the four groups of animals, echocardiography was performed with LOGIQ e Ultrasound (GE Medical System) as previously described [23]. Briefly, the rats were anesthetized with $2 \%$ isoflurane, the chest was shaved, and a layer of acoustic-coupling gel was applied to the thorax. Then the rats were placed in the left lateral decubitus position. M-mode recording of the LV was obtained at the level of the mitral valve in the parasternal view using two-dimensional (2D) echocardiographic guidance in both short and long axis views. Cardiac function and heart dimensions were evaluated by 2D echocardiography on the anesthetized ( $2 \%$ isoflurane) rat. M-mode tracing was used to measure functional parameters such as LV end-diastolic dimension (LVEDD), LV end-systolic dimension (LVESD), LV end-diastolic volume (LVEDV) and LV end-systolic volume (LVESV) were calculated using the above 
primary measurements and accompanying software. The percentage of $\mathrm{LV}$ ejection fraction (EF) was calculated as (LVEDV-LVESV)/LVEDV $\times 100 \%$ and the percentage of LV fractional shortening (FS) was calculated as (LVEDD-LVESD)/LVEDD x 100\%. Echocardiography data were recorded and analyzed blindly to the different treatments.

\section{Myocardial I/R injury model}

After 30 days of nicotine treatment, the rats were subjected to ischemia/reperfusion (I/R) procedure in vivo as described previously [24, 25]. Briefly, rats were anaesthetized with $2 \%$ isoflurane and placed on the RoVent Jr. Small Animal Ventilator (Kent Scientific). Ischemia was induced by an occlusion on left anterior descending (LAD) coronary artery for 45 mins. Reperfusion was initiated after 45 mins of ischemia. Myocardial reperfusion was confirmed by changes in the appearance of the heart from pale to bright red. After 24 hours of reperfusion, some of the rats from each group were anesthetized. Their hearts were rapidly removed and serially sectioned along the short axis in 2-mm-thick sections. To measure the infarct size, the slices were then incubated in $2 \%$ 2,3,5-triphenyltetrazolium chloride (TTC) solution for ten minutes at $37^{\circ} \mathrm{C}$ and immersed in formalin for 30 minutes. Viable tissue stained red, while nonviable tissue remained white. The infarct size and the area of $\mathrm{LV}$ in each slice were analyzed by computerized planimetry (NIH image J software), corrected for the tissue weight, summed for each heart, and expressed as a percentage of the total left ventricle weight.

\section{Masson's trichrome staining}

Masson's trichrome staining is widely used to study cardiac pathologies including cardiac infarction and fibrosis. In the present study, the heart was rapidly excised, rinsed to remove blood. Then, the heart tissues were cryopreserved by using optimal cutting temperature (OCT) tissue medium and sectioned transversely from the basal part to the apex of left ventricle using a cryostat with $10 \mu \mathrm{m}$ thickness. Masson's Trichrome staining (Abcam) was performed according to the manufacture's instruction to quantify infarct scar size (blue staining). The infarct scar size was analyzed by computerized planimetry and expressed as the ratio of scar area to total LV area.

\section{Measurement of superoxide production in heart tissues}

The oxidative fluorescent dye hydroethidium (HE) was used to evaluate superoxide production in situ, as described previously $[19,26]$. Briefly, unfixed frozen heart segments were cut into $10-\mu \mathrm{m}$ thick sections using Leica CM 3050 s cryostat at $-20^{\circ} \mathrm{C}$. The middle slice sections of $\mathrm{LV}$ were used and each tissue slides was incubated with $\mathrm{HE}(5 \mu \mathrm{M})$ at $37^{\circ} \mathrm{C}$ for 30 minutes. Fluorescence in LV sections was obtained with an EVOS FL color imaging system (Life Technologies Corp. Carlsbad, CA). The quantitative analysis of HE fluorescence intensity was analyzed using the NIH Image J software.

\section{Measurement of reactive oxygen species (ROS) production in plasma}

Plasma was collected from the rat after seven days of reperfusion. The total ROS levels in the plasma were measured with the Oxiselect ${ }^{\mathrm{TM}}$ in vitro ROS/RNS assay kit (Cell Biolabs, Inc. San Diego, CA), following the manufacturer's instruction and described previously [19, 27]. Briefly, $50 \mu \mathrm{L}$ of the plasma samples or standard were added to a 96-well plate and mixed with $50 \mu \mathrm{L}$ of catalyst and $100 \mu \mathrm{L}$ of $2^{\prime}, 7^{\prime}$-dichlorodihydrofluorescein diacetate (DCF). After incubation at room temperature for 30 minutes, the fluorescence (Ex480nm/Em530nm) was measured using a Synergy HT Multi-Mode Microplate Reader (Bio-Tek Instruments, Inc., Winooski, VT, USA).

\section{Western immunoblotting}

Protein abundance in heart was measured as previously described [28]. Briefly, the middle slices of LV tissues were isolated and homogenized in a lysis buffer followed by centrifugation at $4^{\circ} \mathrm{C}$ for 20 minutes at $10000 \mathrm{~g}$, and the supernatants were collected. Samples with equal proteins were loaded onto $7.5 \%$ polyacrylamide gel with $0.1 \%$ sodium dodecyl sulfate and separated by electrophoresis at $100 \mathrm{~V}$ for two hours. Proteins were then transferred onto nitrocellulose membranes and incubated with primary antibodies against LC3 (Cell Signaling Technology), Beclin-1 (Cell Signaling Technology), p62 (Cell Signaling Technology), Atg5 (Cell Signaling Technology), GSK3 $\beta$ (Cell Signaling Technology), p-GSK3 $\beta$ (Cell Signaling Technology), respectively. After washing, membranes were incubated with secondary horseradish peroxidase-conjugated antibodies. Proteins were visualized with enhanced chemiluminescence reagents, and blots were exposed to Hyperfilm. Results were quantified with the Kodak electrophoresis documentation and analysis system and Kodak ID image analysis software (Kodak, Rochester, NY). The target protein abundance was normalized to the abundance of GAPDH as a protein loading control.

\section{Statistical analysis}

All data are expressed as the mean \pm SEM obtained from the number (n) of experimental animals given. Differences between the groups were compared by Student's t-test or analysis of variance (ANOVA) using the Graph-Pad Prism software 
(GraphPad Software Version 4, San Diego, CA, USA), where appropriate. For all comparisons, P-values less than 0.05 indicated statistical significance.

\section{Results}

\section{Effects of chronic nicotine exposure on I/R-induced myocardial infarction and heart function}

Chronic nicotine treatment for 30 days had no effect on the body weights of rats (data not shown). Similarly, there was no significant difference in the ratio of heart to body weight between the saline control and nicotine-treated groups (data not shown). As shown in Fig. 1, after 45 minutes of ischemia followed by 24 hours of reperfusion, there was a remarkable increase in myocardial infarction in rats. In the absence of autophagy inhibitor Methyladenine (3-MA), chronic nicotine exposure induced a significant increase in myocardial infarct size as compared with the saline control group under I/R procedure (Fig. 1). However, in the presence of 3-MA (Fig. 1), 3-MA treatment eliminated the difference of infarct size between the saline control and nicotine-treated group under I/R procedure. As shown in Fig. 2, the cardiac scar size after seven days of reperfusion was significantly higher in the nicotine-treated group than the saline control group in the absence of 3-MA $(\mathrm{P}<0.05)$. However, treatment with 3-MA had the trend to attenuate nicotine-mediated cardiac scar size but did not reached significance under I/R procedure (Fig. 2).

Fig. 3 shows typical echocardiographic images at different time periods in both saline control and nicotine-treated groups in the absence and presence of 3-MA treatment. Fig. 4 shows the summary data obtained from the echocardiography analysis. As shown in Fig 4, there were no significant differences of left ventricular end-diastolic dimension (LVEDD), LV end-systolic dimension (LVESD), ejection fraction (EF) and fractional shortening (FS) at baseline and at 30 days of nicotine treatment between the saline control and nicotine-treated groups. However, after ischemia/reperfusion $(I / R)$, the values of LVEDD (Fig. 4A) and LVESD (Fig. 4C) were reperfusion time-dependently increased. In addition, the values of EF (Fig. 4E) and FS (Fig. 4G) were reperfusion time-dependently decreased in nicotine-treated animals as compared to the saline control animals under I/R procedure. More importantly, treatment with autophagy inhibitor, 3-MA, abolished the nicotine-mediated changes of LVEDD (Fig. 4B), LVESD (Fig. 4D), EF (Fig. 4F), and FS (Fig. 4H) in the animals under $\mathrm{I} / \mathrm{R}$ procedure.
A

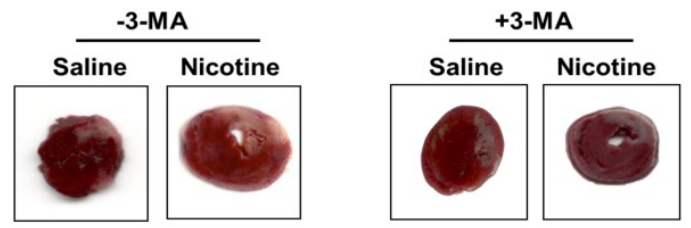

B

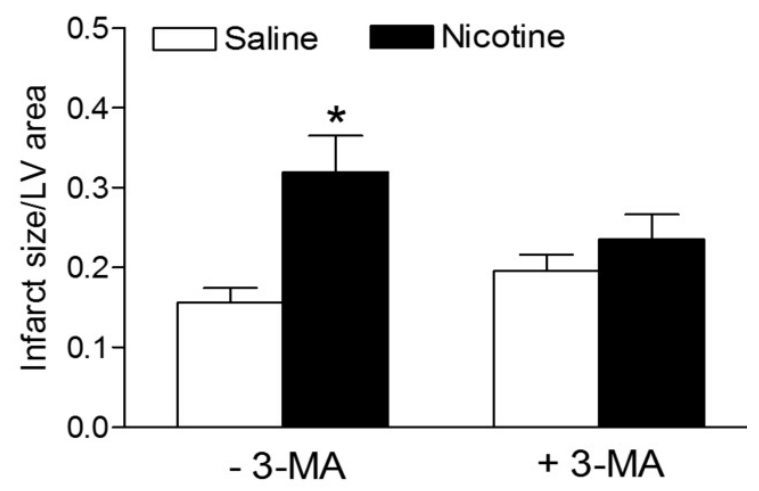

Figure 1. Nicotine exacerbates on ischemia/reperfusion (I/R)-induced myocardial infarct size and 3-MA reduces it. Rats from each group were subjected to $45 \mathrm{~min}$ of heart ischemia followed by 24 hours of reperfusion. After I/R, the hearts were isolated and their infarct sizes in each rat group were determined with $2 \%$ TTC staining (A). The bar graph (B) showing left ventricle infarct size (infarct area/total LV area) in each rat group. Data are means \pm SEM of animals from each group $(n=4-5)$ were analyzed by 2 -way ANOVA. *P $<0.05$ versus saline control.

A

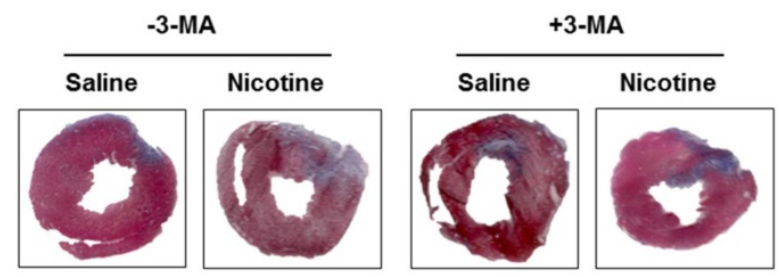

B

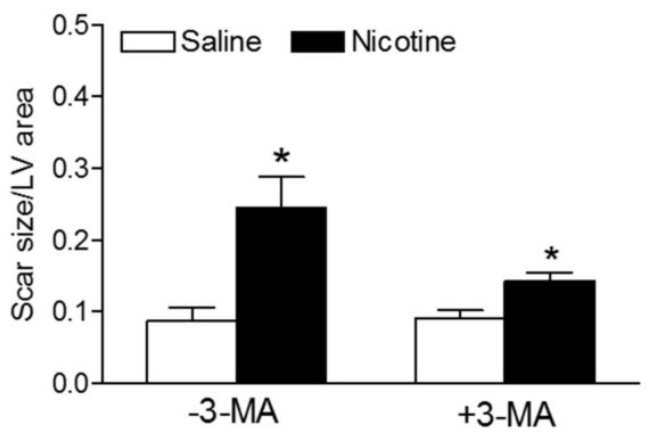

Figure 2. Nicotine enhances ischemia/reperfusion (I/R)-induced relative myocardial scar area. Rats from each group were subjected to $45 \mathrm{~min}$ of heart ischemia followed by seven days of reperfusion. After I/R, the hearts were isolated and their relative scar areas in each rat group were determined with Masson's trichrome staining (A) as described in the Methods section. The bar graph (B) showing left ventricle scar size (ratio of scar area to total LV area) in each rat group. Data are means \pm SEM of animals from each group $(n=4-5)$ were analyzed by 2 -way ANOVA. *P $<0.05$ versus saline control. 

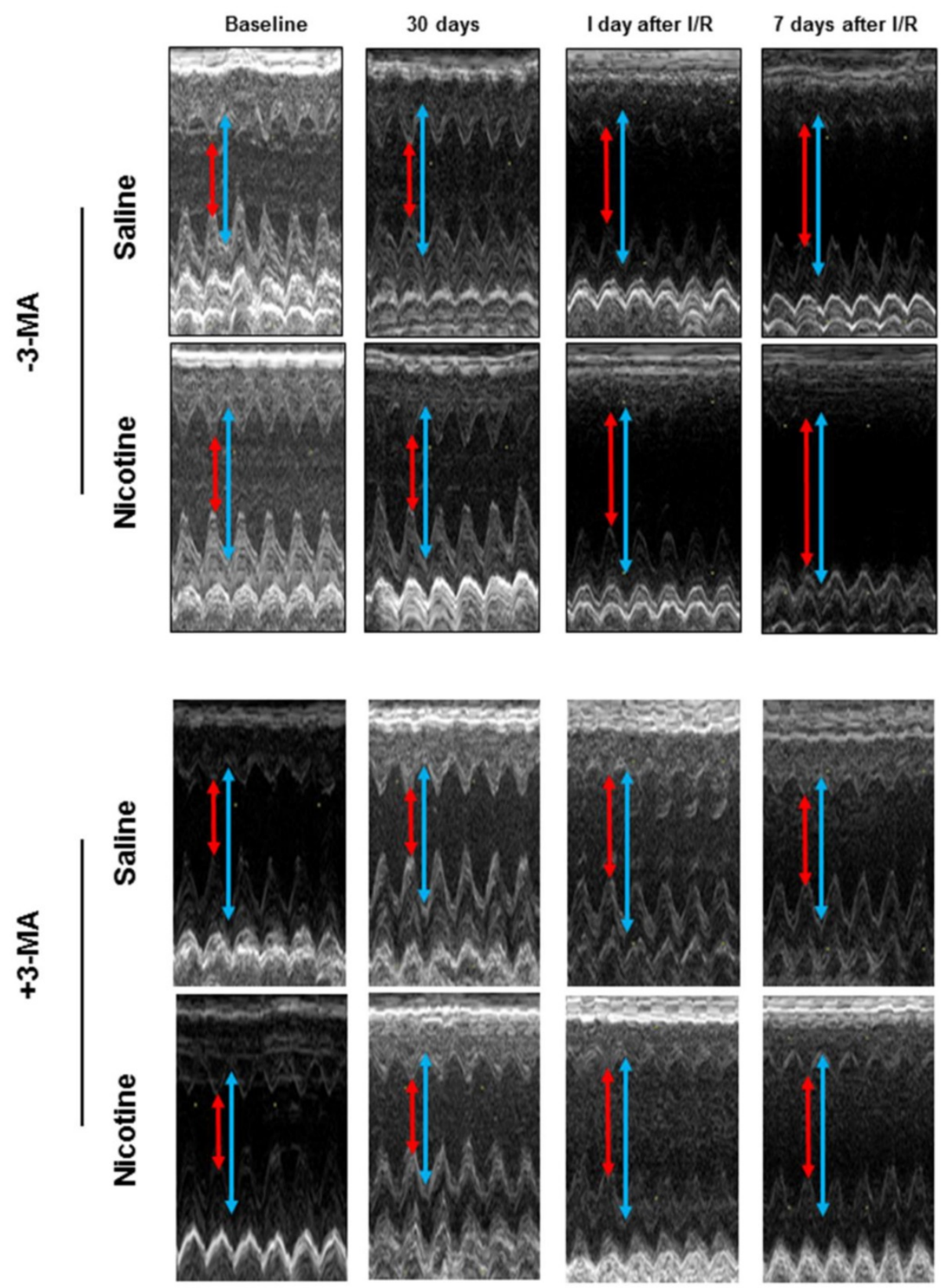

Figure 3. Echocardiographic evaluation of cardiac function. Rats were administered with either saline or nicotine for 30 days. Seven days before I/R surgery, 3-MA or saline was administered to rats as described in Methods section. The representative echocardiographic images were obtained from different time periods including at baseline, 30 days of nicotine treatment, one day after I/R, and seven days after I/R. The red arrow represents LV end-systolic dimension (LVESD) and the blue arrow represents LV end-diastolic dimension (LVEDD).

\section{Effects of chronic nicotine exposure on autophagy-associated biomarkers}

Chronic nicotine exposure significantly enhanced the LC3 II/LC3 I protein expression ratio (Fig. 5A) and p62 protein expression levels (Fig. 5C) in the LV tissues as compared with saline control group in the absence of 3-MA under I/R procedure. However, in the presence of 3-MA, the ratio of LC3 II/LC3 I protein (Fig 5B) and the levels of p62 protein (Fig. 5D) in the LV tissues were not significantly different between the saline control and nicotine-treated rats under I/R procedure. Similarly, the protein levels of Beclin 1 (Fig. 6A) and Atg5 (Fig. 6C) in the LV tissues were higher in the nicotinetreated rats than those in the saline control group, and 3-MA treatment also eliminated these differences between the saline control and nicotine exposed groups under I/ R procedure (Fig. $6 \mathrm{~B}$ and $6 \mathrm{D}$ ).

\section{Effects of chronic nicotine exposure on reactive oxygen species (ROS) production}

To determine whether chronic nicotine exposure causes oxidative damage in the heart, production of superoxide anion $\left(\mathrm{O}_{2}^{-}\right)$was assessed by the oxidative fluorescent dye HE. As shown in Fig. 7A and B, 
chronic nicotine exposure increased the production of $\mathrm{O}_{2}{ }^{-}$in the $\mathrm{LV}$ tissues as compared with the saline control groups in the absence of 3-MA under I/R procedure. Interestingly, 3-MA treatment did not affect nicotine-mediated increase in $\mathrm{O}_{2}$ - production under I/ $\mathrm{R}$ procedure. In addition, we determined the

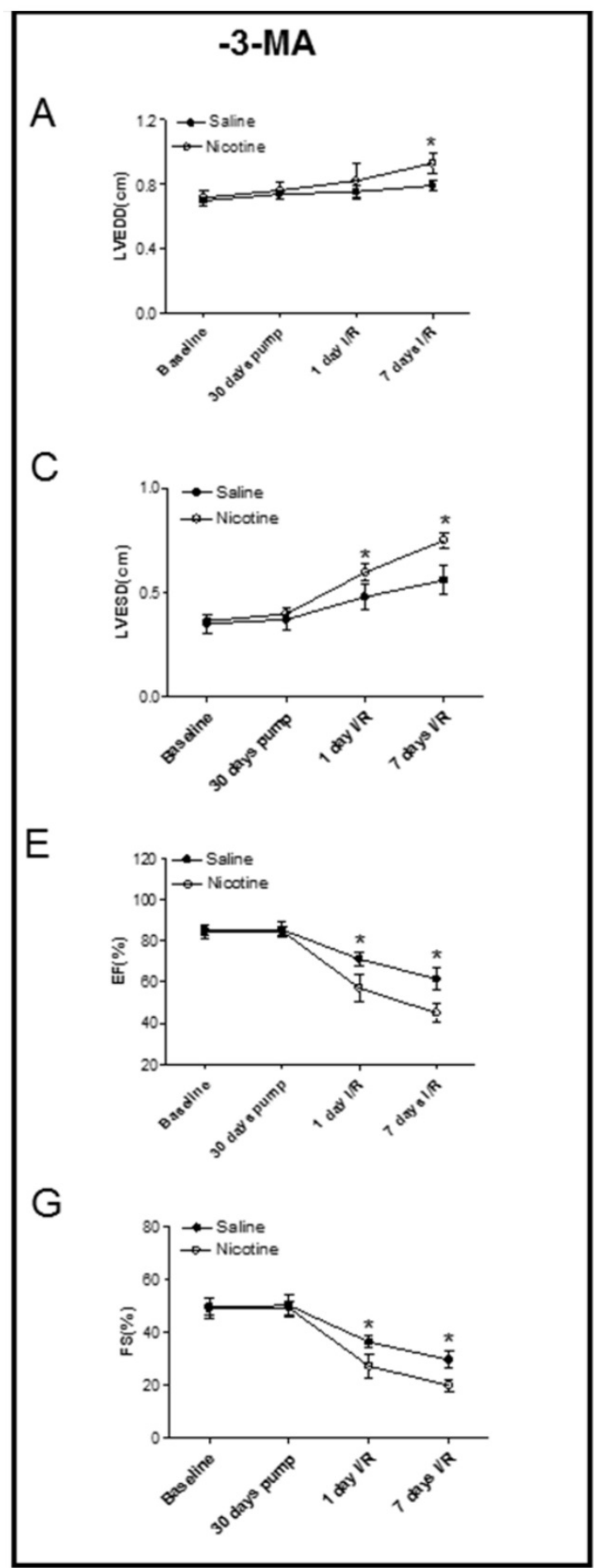

ROS production in the plasma using a ROS assay kit under I/R procedure. As shown in Fig. $7 \mathrm{C}$, the plasma ROS levels in the nicotine-treated animals were substantially higher than those in the saline control animals, and 3-MA treatment had no effect on nicotine-mediated ROS production in the plasma.

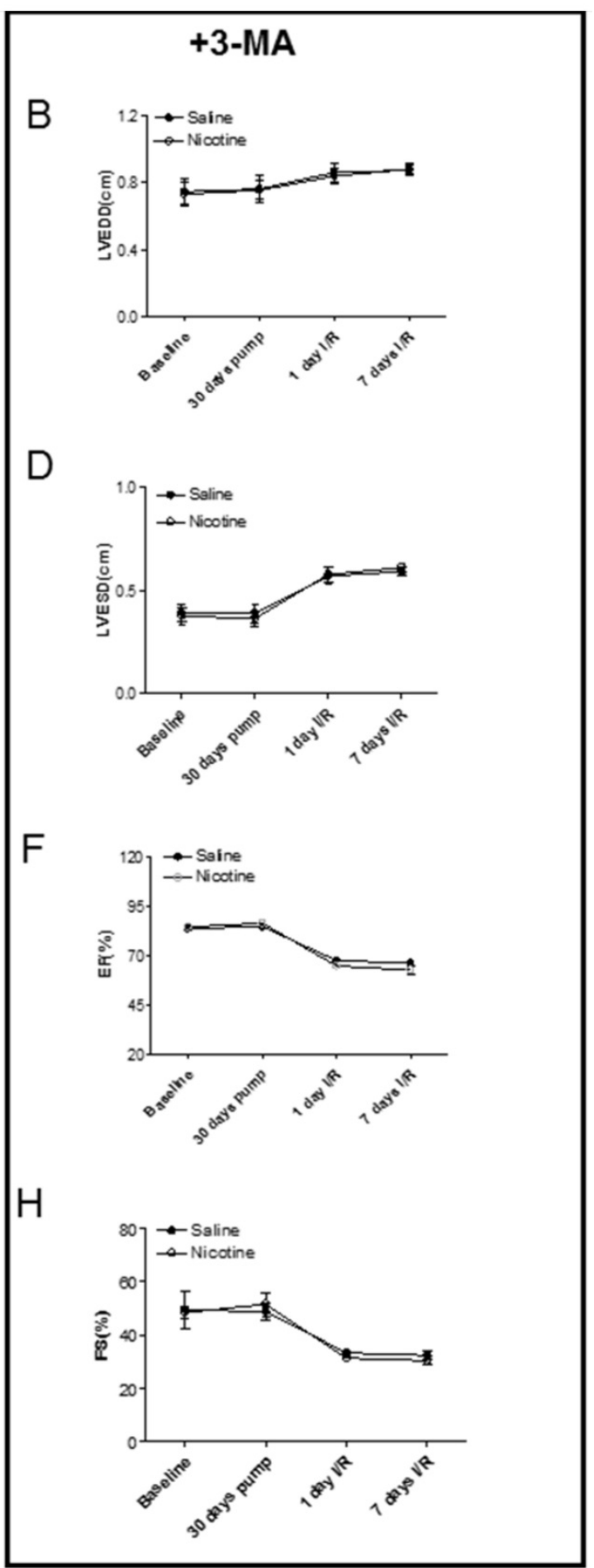

Figure 4. Effects of nicotine exposure on cardiac function before and after I/R. The echocardiographic data of both the saline control and nicotine-treated groups were examined at following time periods: before nicotine treatment (baseline), 30 days of nicotine treatment, one day after I/R, and seven days after I/R. The summary data include: left ventricular end-diastolic dimension (LVEDD) in the absence (A) and in the presence of (B) 3-MA treatment; left ventricular end-systolic dimension (LVESD) in the absence (C) and in the presence of (D) 3-MA treatment; percent of ejection fraction (EF\%) in the absence (E) and in the presence of (F) 3-MA treatment; percent of fractional shortening (FS\%) in the absence $(\mathbf{G})$ and in the presence of $(\mathbf{H})$ 3-MA treatment. Data are means \pm SEM of animals from each group ( $\mathrm{n}=4-5)$ were analyzed by 2-way ANOVA. $* \mathrm{P}<0.05$ versus saline control. 


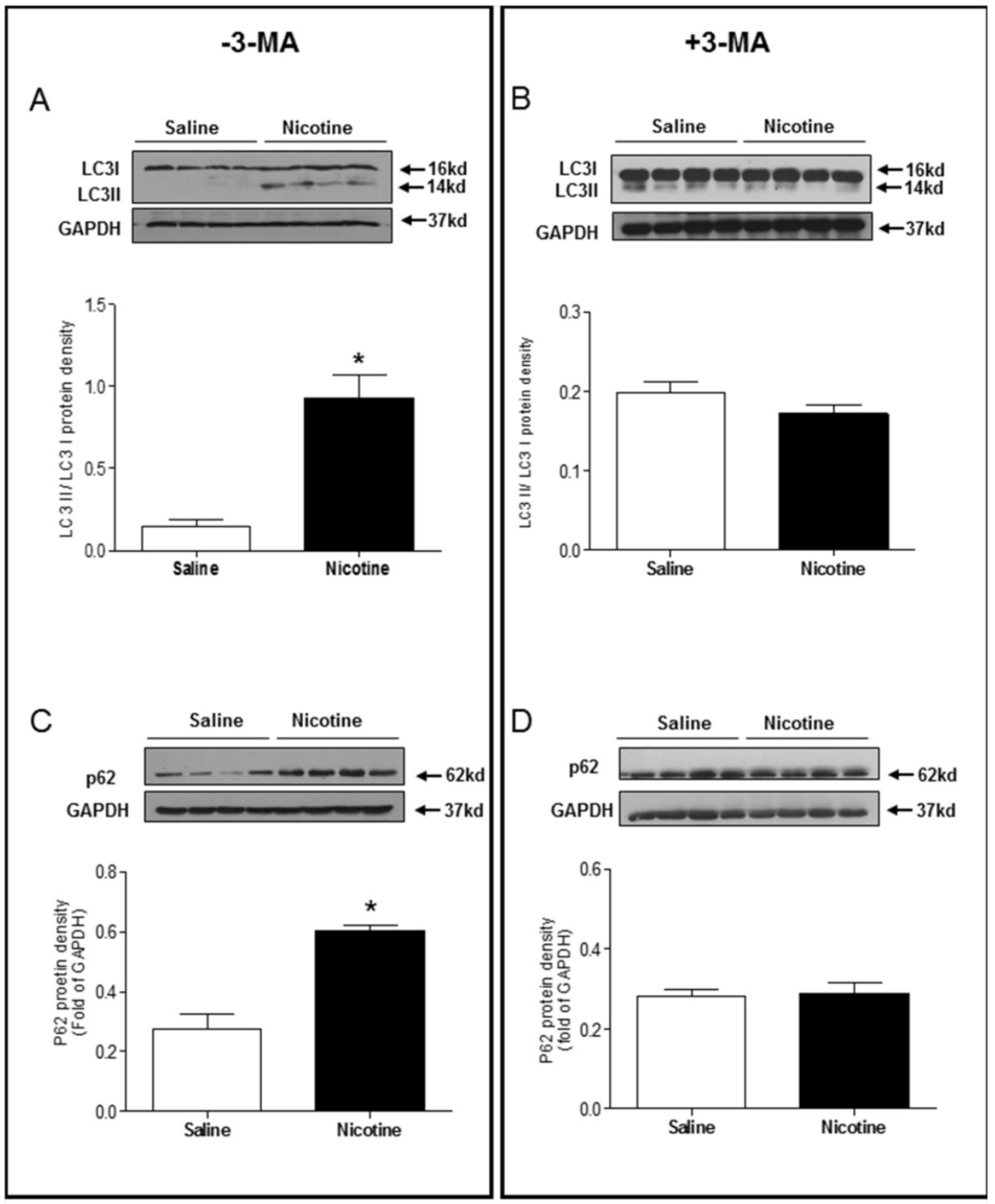

Figure 5. Effects of nicotine exposure on the protein expressions of LC3 II/LC3 I and p62. Rats from each group were subjected to 45 minutes of heart ischemia and seven days of reperfusion. After I/R, the hearts were isolated and the protein abundance in the left ventricle tissue was determined by Western blot analysis. LC3 II/LC3 I protein density in the absence of 3-MA (A) and in the presence of 3-MA (B) treatment. p62 protein density in the absence of 3-MA (C) and in the presence of 3-MA (D) treatment. Data are means \pm SEM ( $n=4$ animals/groups). $* P<0.05$ vs. control, as determined by Student's t-test.

Effects of chronic nicotine exposure on cardiac glycogen synthase kinase 3 beta (GSK3ß) activity

GSK3 $\beta$ is a master regulator of cardiac cell growth and death, which is highly involved in the setting of heart ischemic injury. To investigate the role of GSK3 $\beta$ signaling in mediating cardiac dysfunction in response to nicotine exposure, the phosphorylation levels of GSK3 $\beta$ at serine 9 and total GSK3 $\beta$ protein expression were determined by Western blot analysis.
As shown in Fig. 8, chronic nicotine exposure had no significant effect on GSK3 $\beta$ protein expression, but enhanced the ratio of serine 9 phosphorylation of GSK3 $\beta$ to total GSK3 $\beta$ protein expression in the LV tissues as compared with the control groups under I/R procedure. In addition, 3-MA treatment eliminated the difference of the ratio of serine 9 phosphorylation of GSK3 $\beta$ to total GSK3 $\beta$ protein expression between the control and nicotine-treated animals under I/R procedure. 


\section{Discussion}

Epidemiological and animal studies have shown that tobacco smoking increases the risk of ischemic heart disease [3, 29], and suggest that nicotine contributes to myocardial ischemia [3]. The present study provides further direct evidence that chronic nicotine exposure increases myocardial infarct size and heart dysfunction in a rat model of ischemia/reperfusion. The major findings in the present study are: (1) chronic nicotine exposure increased cardiac I/R injury and resulted in cardiac dysfunction in vivo, which was associated with a significant increase in autophagy biomarkers, oxidative stress-related ROS, and ischemia-related protein GSK3 $\beta$; (2) inhibition of autophagy with an autophagy inhibitor normalized the aberrant autophagy level and reversed the increased vulnerability to heart I/R injury and dysfunction. These findings suggest a potentially novel therapeutic strategy of anti-autophagy for the treatment of smoking/nicotine-mediated heart ischemic disease.

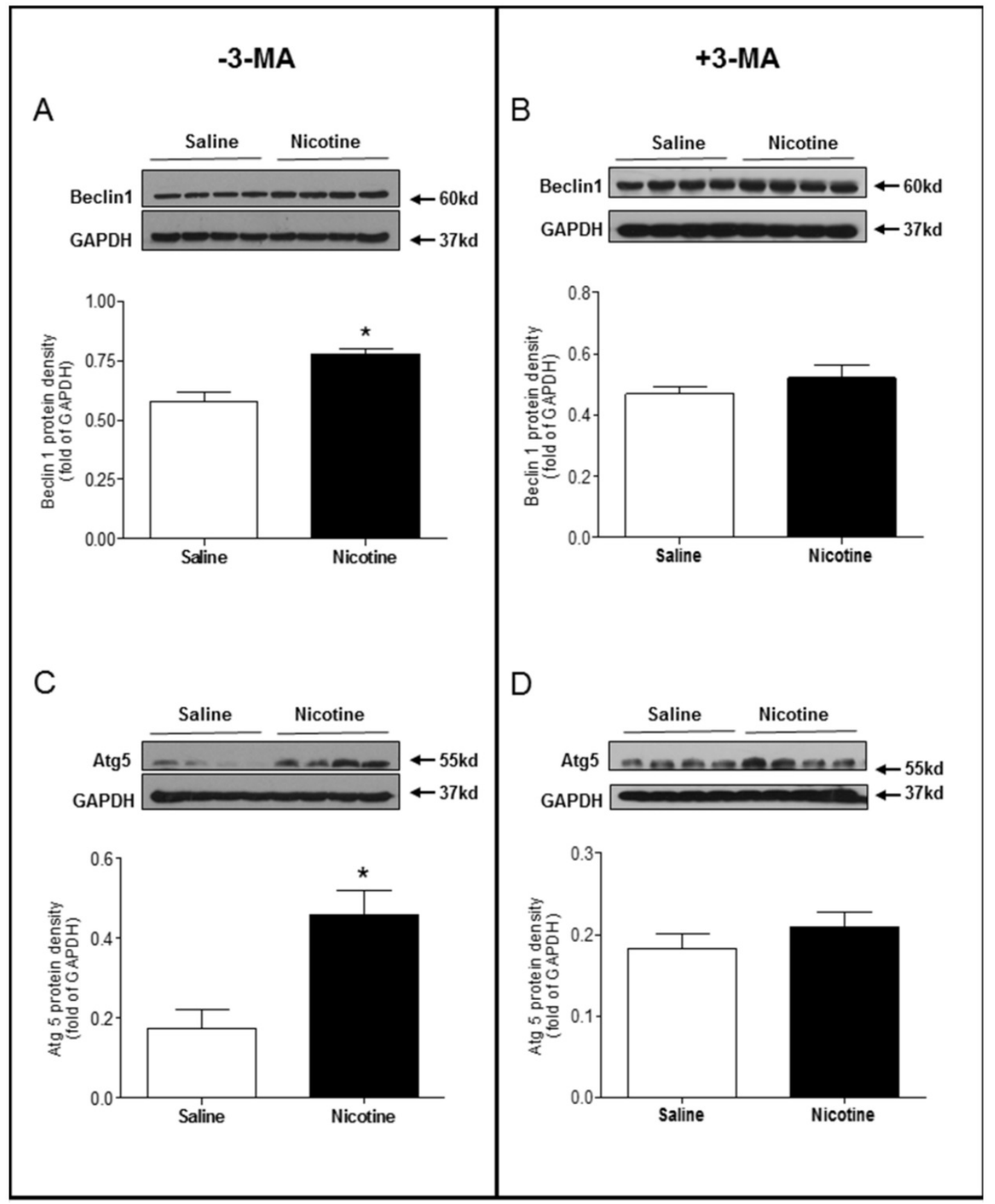

Figure 6. Effects of nicotine exposure on the protein expressions of Beclin 1 and Atg5. Rats from each group were subjected to 45 minutes of heart ischemia and seven days of reperfusion. After I/R, the hearts were isolated and the protein abundance in the left ventricle tissue was determined by Western blot analysis. Beclin 1 protein density in the absence of 3-MA (A) and in the presence of 3-MA (B) treatment. Atg5 protein density in the absence of 3-MA (C) and in the presence of 3-MA (D) treatment. Data are means \pm SEM ( $n=4$ animals/groups). $* P<0.05$ vs. control, as determined by Student's t-test. 
-3-MA

A

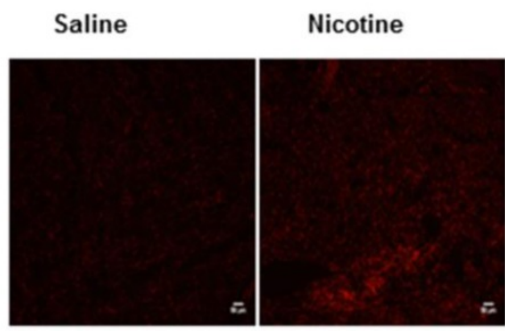

+3-MA

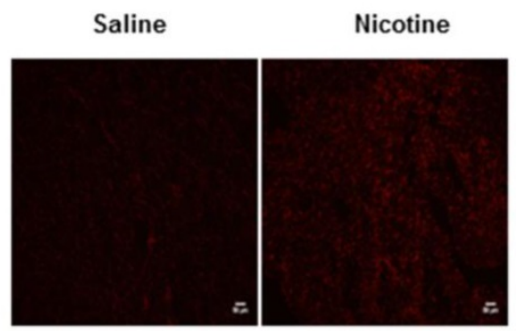

B

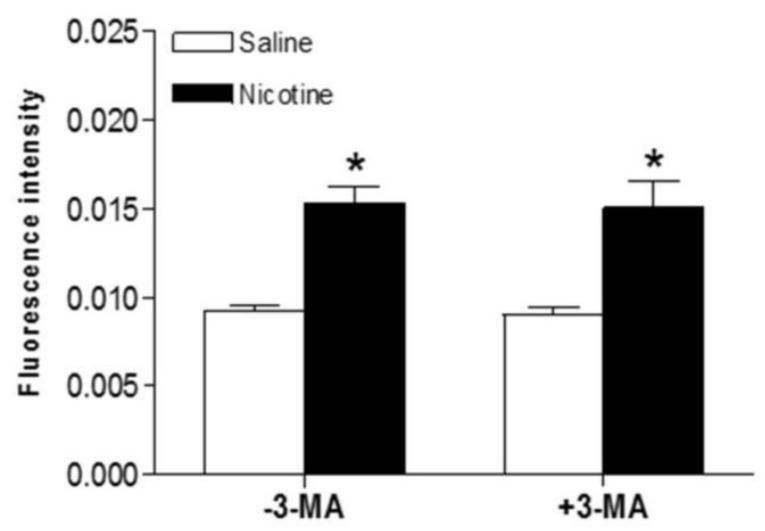

C

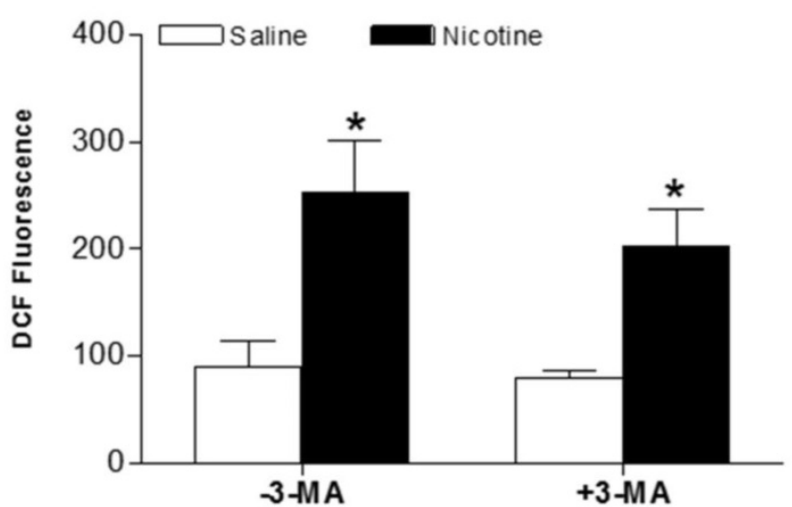

Figure 7. Effects of nicotine exposure on cardiac and plasma ROS production. Rats from each group were subjected to 45 minutes of heart ischemia and seven days of reperfusion. After I/R, plasma from each rat was collected and LV segments were cut into 10- $\mu \mathrm{m}$ thick sections as described in Methods section. HE fluorescence from each LV section was used to image (A) and analyze ROS production (B) in situ in the absence and presence of 3-MA. Plasma ROS levels were measured using in vitro ROS/RNS assay kit (C). Data are means \pm SEM of tissue from 4 animals from each group. Scale bar is $50 \mu \mathrm{m}$. $* \mathrm{P}<0.05$ versus saline control, as determined by Student's t-test.

Our data indicated that the ratio of heart weight to body weight was not changed between the control and nicotine-treated groups. In addition, although we did not measure the ratio of heart weight/body weight after seven days of reperfusion, our echocardiographic observations indicated that there were no differences of LVPWd (data not shown) between the nicotine-treated and control groups. These data suggest that nicotine does not induce cardiac hypertrophy at both resting and I/R-challenged condition. The present study showed that chronic nicotine exposure had no significant effect on pre-ischemic baseline values of heart function determined by echocardiography but significantly increased LV myocardial infarct size and impaired LV function after ischemia/reperfusion in rats. The data suggests that chronic nicotine exposure does not affect heart function at normal physiologic conditions but alters the heart function when it encounters an ischemic stress challenge. Previous studies in human and animal models have reported that cigarette smoking is one of the top risk factors of ischemic heart disease $[3,4,6,29]$. Furthermore, there were positive relations between smoking-mediated myocardial infarct size and nicotine concentration in the animal model [3]. The present findings that chronic nicotine exposure enhanced I/R-induced myocardial infarct and scar size, suggest that nicotine 
is a key factor in cigarette smoking-induced cardiac infarction and heart dysfunction. As a ganglionic agonist, nicotine can bind the nicotinic Ach receptor and stimulate neurotransmitter, catecholamine release. Previous studies have demonstrated that cigarette smoking significantly increases plasma norepinephrine and epinephrine levels, which are stimulated by nicotine [5, 6]. Increased circulating concentrations of catecholamines enhance the pulse rate, blood pressure, and ischemic heart injury $[5,6$, 28]. Therefore, it is likely that cigarette smoking/nicotine exposure-associated increments in ischemic heart infarction are mediated through adrenergic mechanisms by releasing catecholamine. Indeed, our previous studies in a similar pregnant rat model have demonstrated that maternal nicotine exposure during pregnancy enhances norepinephrine levels in the fetal heart, resulting in development of heart ischemia-sensitive phenotype [30].

The molecular mechanisms underlying nicotine-mediated increase in ischemic injury and heart dysfunction are still largely unknown. In the present study, we observed that chronic nicotine exposure enhanced autophagy biomarkers (such as LC3II, p62, Beclin 1, and Atg 5) protein abundance in cardiac tissues. Consistent with our observation, other studies have shown that nicotine exposure increases autophagy activation in human fibroblast cells [31], human cancer colon cells [32], and bronchial epithelial cells [13]. These findings suggest that nicotine is one of the key activators of autophagy. Growing evidence has shown an important role of autophagy in the pathophysiological process of ischemic heart injury [33]. However, whether autophagy serves a beneficial or detrimental role in the myocardium depends on the level of autophagy activation and the context in which it is induced $[8,10,11]$. It has been reported that upregulation of autophagy in the setting of mild ischemia provides a protective effect that promotes functional recovery of the heart. However, there is also strong evidence that excessive autophagy contributes to myocyte death and heart dysfunction [11]. Our present findings that treatment with 3-MA significantly inhibited nicotine-mediated autophagy and eliminated the difference of I/R-induced cardiac injury and dysfunction between the saline control and nicotine-treated rats, suggest that nicotine-mediated heightened autophagy is one of the key molecular mechanisms contributing to ischemic heart injury and dysfunction. Consistent with our findings, a previous study has shown that enhanced autophagy leads to accumulation of autophagosomes and cardiac contractile dysfunction, and inhibition of autophagy with 3-MA treatment prevents heart injury [34]. In addition, other studies in rat primary cardiac myocytes in vitro have demonstrated that treatment of 3-MA or knockdown of Beclin 1 inhibits ischemia/ reperfusion-induced autophagy, leading to reduced cardiac injury and enhanced cardiac myocyte survival [35]. In contrast to these finding, our current data (Fig. 1 and 2) indicated that in vivo treatment of 3-MA had no effect on I/R-induced heart injury in saline control animals. This discrepant effect of 3-MA between in vitro cardiac myocytes and in vivo heart may suggest
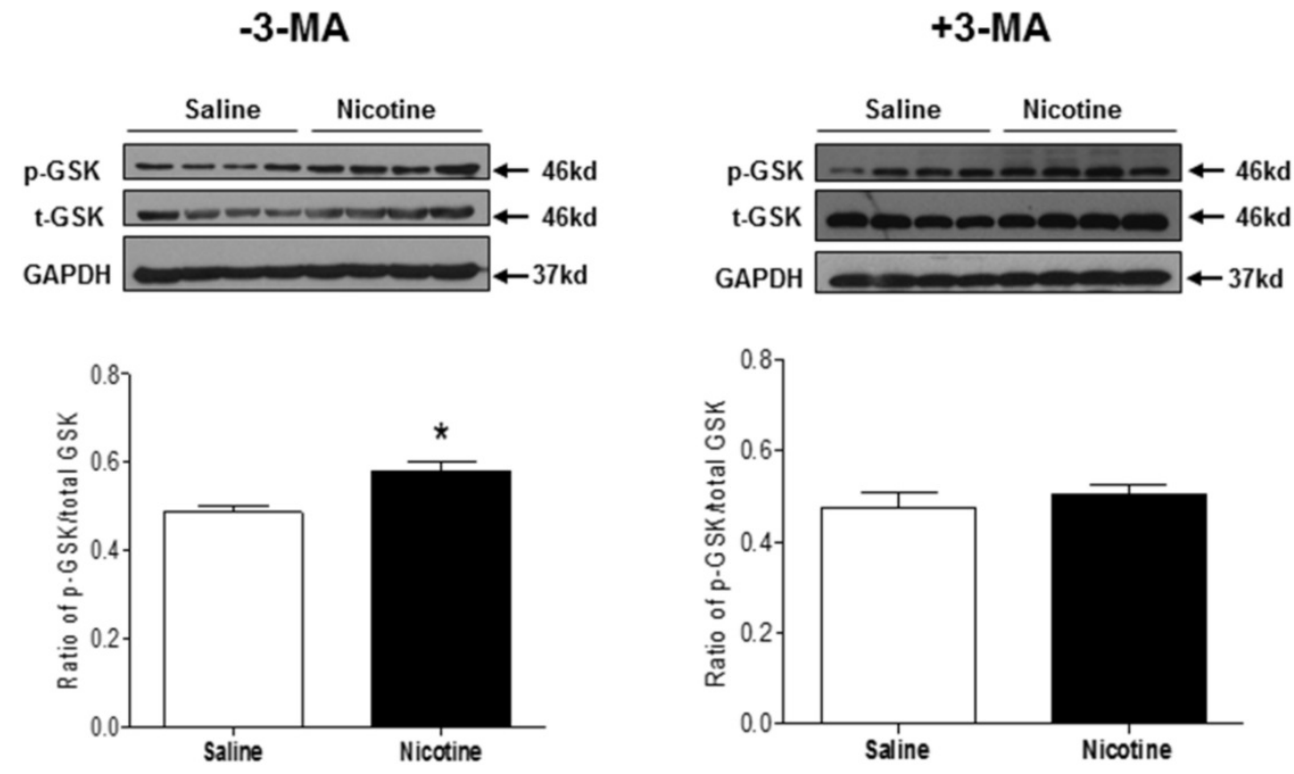

Figure 8. Effects of nicotine exposure on the protein expressions of total GSK3 $\beta$ and phosphorylation of GSK3 $\beta$ (ser9). Rats from each group were subjected to 45 minutes of heart ischemia and seven days of reperfusion. After I/R, the hearts were isolated. The protein abundances of p-GSK3 3 and total GSK3 $\beta$ in the left ventricle tissues were determined in the absence of $3-M A$ and in the presence of 3-MA. Data are means \pm SEM ( $n=4$ animals/groups). $* P<0.05$ vs. control, as determined by Student's t-test. 
that basal autophagy dose not play a key role in I/R-induced heart injury in animals at physiologic condition. However, our present findings that that treatment with 3-MA significantly inhibited I/Rinduced heart infarct size in nicotine-treated animals and eliminated the difference of I/R-induced cardiac infarct size and dysfunction between the saline control and nicotine-treated rats, suggest that the excess of autophagy plays an important role in nicotine-mediated I/R-induced heart ischemic injury and dysfunction. Taken together, our current findings with others suggest a causal effect of autophagy in the setting of ischemic heart injury and dysfunction under pathologic condition. In addition, we need keep in mind that autophagy signaling pathway may be not the only signaling pathway involved nicotinemediated cardiac dysfunction, other signaling pathways such as apoptosis may also be involved.

The process of autophagy is complex and mainly comprised of the following stages: initiation, nucleation, expansion, maturation, and degradation. Numerous autophagy-related (Atg) genes and signaling molecules have been identified and are sequentially activated to regulate different stages of autophagy. There are three classes of PtdIns3K and phosphatidylinositol 3-Kinases (PI3Ks) involved in the regulation of autophagy. Class I PI3K responds to growth factor signaling and produces PtdIns $(3,4,5) \mathrm{P} 3$ that negatively regulates autophagy via the AKT-mTOR complex, whereas the class III PI3K, as well as class II PI3K, contributes to the initiation and progression of autophagy. Beclin 1 is one of the major genes involved in the class III PI3K complex signaling [36]. The formation of autophagosome is often initiated with Beclin1 as an internal stimulus followed by several autophagy related proteins including Atg5 [37]. In the present study, our data indicated that nicotine exposure enhanced the protein levels of Beclin1 and Atg5 in the LV tissues, which were reversed by the treatment of 3-MA. Consistent with our findings, previous studies demonstrated that cigarette smoking induces autophagy via increasing Beclin1 and Atg5 gene expressions [38]. These data suggest that activation of the class III PI3K signaling pathway is one of the important mechanisms underlying nicotine-induced cardiac autophagy. In addition to the regulation of class III PI3K pathway, our present findings that nicotine exposure significantly increased p62 protein levels in ischemic heart tissues, which was abrogated by 3-MA treatment, suggest that p62 also play a key role in nicotine-mediated autophagy. Similarly, previous studies have demonstrated that nicotine increases co-expression of impaired autophagy marker, SQSTM1/p62 and ubiquitin [13]. It is well known that ubiquitinated cargo including injury organelles and potentially toxic protein aggregates is delivered to autophagosomes by the receptor protein p62 [39, 40]. The interaction between autophagosomes and SQSTM1/p62 has been observed in smokers' alveolar macrophages [41]. The formation of autophagosome is often initiated with Beclin1 as an internal stimulus followed by several autophagy related proteins including Atg5 [37]. Thus, we speculate that Beclin 1/Atg5/p62/LC3II signaling may be one of the major signaling pathways underlying nicotine-mediated autophagy and ischemic heart injury.

It has been shown that cigarette smoking increases the sensitivity of hearts to ischemia/ reperfusion injury associated with increased production of oxygen free radicals [42]. Consistent with this study, our present study also showed an increase in ROS production in both plasma and cardiac tissue of the rat exposed to nicotine. Furthermore, our previous studies have demonstrated that inhibition of ROS reverses perinatal nicotinemediated ischemic heart injury and heart dysfunction [43]. These studies suggest that oxidative stress plays a key role in the aberrant development of heart ischemia-sensitive phenotype in response to cigarette smoking/nicotine exposure. The relationship between ROS and autophagy has been demonstrated in several other systems [44, 45], but the cause-and-effect relation is controversial. For example, amino acid deprivation induces the formation of ROS in mitochondria in a Class III PI3K-dependent manner [45]. On the contrary, p62 overexpression increases ROS and accelerates tumorigenesis [46]. Although molecular mechanisms underlying nicotine-induced excessive autophagy in the heart are not fully understood, previous studies have shown that stress-induced ROS is essential for the induction of autophagy [45]. We speculate that nicotine-mediated ROS release may be one of the key mechanism underlying nicotine-induced excessive autophagy. Indeed, our current study found that 3-MA treatment did not affect nicotine-mediated ROS production. Consistent with our finding, previous studies have also demonstrated that 3-MA has no effect on ROS production in starvation-induced or dexamethasoneinduced autophagy [47]. These findings suggest that ROS is the up-stream signaling of autophagy in our animal model. Future studies are needed to employ a specific ROS scavenger or antioxidant to confirm whether the heightened ROS is one of the key mechanisms underlying nicotine-induced autophagy.

GSK3 $\beta$ gene plays a significant role in embryonic cardiomyocyte development. Recent studies demonstrate a functional role of GSK3 $\beta$ in negative modulation of cardiac hypertrophy and in the setting 
of cardiac ischemic disease [48-50]. GSK3 $\beta$ is a unique protein kinase. The dephosphorylated GSK3 $\beta$ is in active form, but GSK3 $\beta$ will be inactivated when it is phosphorylated at the serine 9 residue by other factors $[51,52]$. In present study, we demonstrated that chronic nicotine exposure enhanced the phosphorylation levels of GSK3 $\beta$ at the serine 9 residue as compared to the saline controls. This suggests that the enhanced phosphorylation of GSK3 $\beta$ may lead to a decrease in cardiac GSK3 $\beta$ activity. In addition, our present data that treatment of 3-MA eliminated the difference of the phosphorylation level of GSK3 $\beta$ between the saline control and nicotine-treated animals, suggests that the enhanced phosphorylation of GSK3 $\beta$ is at least partially regulated through an autophagy-dependent signaling pathway.

In summary, the present study provides new evidence that excess autophagy is one of the key molecular signaling linkers between nicotine exposure and the development of the heart ischemic-sensitive phenotype. Our data indicated that nicotine exposure stimulated oxidative stress and enhanced cardiac autophagy biomarkers, resulting in increased ischemic heart infarct size and heart dysfunction. Of importance, inhibition of autophagy via 3-MA rescued nicotine-mediated pathological effects and heart dysfunctions. These findings not only provide novel evidence regarding the molecular mechanisms underlying nicotine-induced ischemic heart injury and dysfunction, but also suggest a potential therapeutic molecular target of autophagy involved in the setting of ischemic heart disease.

\section{Future studies}

In our present study we have demonstrated that chronic nicotine exposure enhances I/R-induced heart dysfunction associated with an excessive autophagy flux. However, one of the limitations for this study is unknown whether the induction of the autophagy proteins is directly regulated by nicotine exposure or I/R procedure. Therefore, in our future study we should measure the basal levels of autophagy influx in the control and nicotine-treated animals to see whether nicotine-mediated pre-existed increase of autophagy in the heart would exacerbate the ischemic/reperfusion injury. While infarct size and cardiac function are good indicators of myocardial ischemia/reperfusion injury, other indicators such as Troponin I concentration can further support the claim. Thus, in our future studies, we will investigate whether nicotine exposure affects Troponin I expression in response to I/R. It's well known that 3-MA is a selective autophagy/PI3K inhibitor. However, 3-MA could induce potential non-specific effects on heart function. It depends on the patho-physiologic condition of the animals and dosage of 3-MA. For instance, previous studies have reported that 3-MA has dual role in modulation of autophagy and induces an autophagic promotion effect under nutrient-rich condition [53]. Moreover, 3-MA can inhibit tumor metastasis in an autophagy-independent manner [54]. Furthermore, 3-MA has an anti-inflammatory effect in atherosclerosis [55]. Therefore, further investigation is needed to unmask the diverse regulations of 3-MA on nicotine-mediated heart I/R injury and dysfunction.

\section{Abbreviations}

3-MA: 3-methyladenine; I/R: ischemia/reperfusion; LC3: microtubule-associated protein light chain 3; P62: sequestosome 1; Atg5: autophagy related 5; ROS: reactive oxygen species; RNS: reactive nitrogen species; GSK3 $\beta$ : glycogen synthase kinase 3 beta; LV: left ventricle; LVEDD: left ventricular end-diastolic dimension; LVESD: left ventricular end-systolic dimension; LVEDV: left ventricular end-diastolic volume; LVESV: left ventricular end-systolic volume; LVPWd: left ventricular posterior wall thickness at end-diastole; EF: ejection fraction; FS: fractional shortening; LAD: left anterior descending artery; TTC: 2,3,5-triphenyltetrazolium chloride; OCT: optimal cutting temperature; HE: hydroethidium; DCF: 2',7'-dichlorodihydrofluorescein diacetate; GAPDH: glyceraldehyde 3-phosphate dehydrogenase; ANOVA: analysis of variance; PI3K: phosphatidylinositol 3-kinases; AKT: protein kinas B; mTOR: mammalian target of rapamycin.

\section{Acknowledgments}

This work was supported by National Institutes of Health Grants HL135623 (DX \& MS), DA041492 (DX), and HD088039 (DX). This project was partially supported by funds provided by The Regents of the University of California, Research Grants Program Office, Tobacco Related Disease Research Program (TRDRP) grant \# T29IR0437 (DX). The funders had no role in study design, data collection and analysis, decision to publish, or preparation of the manuscript.

\section{Competing Interests}

The authors have declared that no competing interest exists.

\section{References}

1. Kannel WB, Higgins M. Smoking and hypertension as predictors of cardiovascular risk in population studies. J Hypertens Suppl. 1990; 8: S3-8.

2. Peto R, Boreham J, Lopez AD, Thun M, Heath C. Mortality from tobacco in developed countries: indirect estimation from national vital statistics. Lancet. 1992; 339: 1268-78. 
3. Zhu BQ, Sun YP, Sievers RE, Glantz SA, Parmley WW, Wolfe CL. Exposure to environmental tobacco smoke increases myocardial infarct size in rats. Circulation. 1994; 89: 1282-90.

4. Zhu BQ, Sun YP, Sievers RE, Shuman JL, Glantz SA, Chatterjee K, Parmley WW, Wolfe CL. L-arginine decreases infarct size in rats exposed to environmental tobacco smoke. Am Heart J. 1996; 132: 91-100.

5. Cryer PE, Haymond MW, Santiago JV, Shah SD. Norepinepinephrine and epinephrine release and adrenergic mediation of smoking associated hemodynamic and metabolic events. N Engl J Med. 1976; 295: 573-7.

6. Schror K, Zimmermann KC, Tannhauser R. Augmented myocardial ischemia by nicotine-mechanisms and their possible significance. $\mathrm{Br} \mathrm{J}$ Pharmacol. 1998; 125: 79-86.

7. Winniford MD, Wheelan KR, Kremers MS, Ugolini V, van den Berg E Jr, Niggemann EH, Jansen DE, Hillis LD. Smoking induced coronary vasoconstriction in patients with atherosclerotic coronary artery disease: evidence for adrenergically mediated alteration in coronary artery tone. Circulation. 1986; 73: 662-7.

8. Ma S, Wang Y, Chen Y, Cao F. The role of the autophagy in myocardial ischemia/reperfusion injury. Biochim Biophys Acta. 2015; 1852: 271-6.

9. Maron BJ, Roberts WC, Arad M, Haas TS, Spirito P, Wright GB, Almquist AK, Baffa JM, Saul JP, Ho CY, Seidman J, Seidman CE. Clinical outcome and phenotypic expression in LAMP2 cardiomyopathy. JAMA. 2009; 301: 1253-9.

10. Lavandero S, Chiong M, Rothermel BA, Hill JA. Autophagy cardiovascular biology. J Clin Invest. 2015; 125: 55-64.

11. Sciarretta S, Hariharan N, Monden Y, Zablocki D, Sadoshima J. Is autophagy in response to ischemia and reperfusion protective or detrimental for the heart? Pediatr Cardiol. 2011; 32: 275-81.

12. Matsui $Y$, Kyoi S, Takagi H, Hsu CP, Hariharan N, Ago T, Vatner SF, Sadoshima J. Molecular mechanisms and physiological significance of autophagy during myocardial ischemia and reperfusion. Autophagy. 2008; 4: 409-15.

13. Bodas M, Van Westphal C, Carpenter-Thompson R, KMohanty D, Vij N. Nicotine exposure induces bronchial epithelial cell apoptosis and senescence via ROS mediated autophagy-impairment. Free Radic Biol Med. 2016; 97: 441-53.

14. Gannon AM, Stampfli MR, Foster WG. Cigarette smoke exposure elicits increased autophagy and dysregulation of mitochondrial dynamics in murine granulosa cells. Biol Reprod. 2013; 88: 63.

15. Liu S, Sun Y, Li Z. Resveratrol protects Leydig cells from nicotine-induced oxidative damage through enhanced autophagy. Clin Exp Pharmacol Physiol. 2018; 45: 573-80.

16. Vij N, Chandramani-Shivalingappa P, Van Westphal C, Hole R, Bodas M. Cigarette smoke-induced autophagy impairment accelerates lung aging, COPD-emphysema exacerbations and pathogenesis. Am J Physiol Cell Physiol. 2018; 314: C73-C87.

17. Li Y, Xiao D, Dasgupta C, Xiong F, Tong W, Yang S, Zhang L. Perinatal nicotine exposure increases vulnerability of hypoxic-ischemic brain injury in neonatal rats: Role of angiotensin II receptors. Stroke. 2012; 43: 2483-90.

18. Li Y, Xiao D, Yang S, Zhang L. Promoter methylation represses AT2R gene and increases brain hypoxic-ischemic injury in neonatal rats. Neurobiol Dis. 2013; 60: 32-8

19. Xiao D, Huang $X$, Yang $S$, Zhang L. Antenatal nicotine induces heightened oxidative stress and vascular dysfunction in rat offspring. $\mathrm{Br}$ J Pharmacol. 2011; 164: 1400-9.

20. Slotkin TA. Fetal nicotine or cocaine exposure: which one is worse? J Pharmacol Exp Ther. 1998; 285: 931-45

21. Maeda H, Nagai H, Takemura G, Shintani-Ishida K, Komatsu M, Ogura S, Shirai M, Kuwahira I, Yoshida K. Intermittent-hypoxia induced autophagy attenuates contractile dysfunction and myocardial injury in rat heart. Biochim Biophys Acta. 2013; 1832: 1159-66.

22. Zhang D, Qiu W, Wang P, Zhang P, Zhang F, Wang P, Sun Y. Autophagy can alleviate severe burn-induced damage to the intestinal tract in mice. Surgery. 2017; 162: 408-17.

23. Zhang P, Lv J, Li Y, Zhang L, Xiao D. Neonatal Lipopolysaccharide Exposure Gender-Dependently Increases Heart Susceptibility to Ischemia/Reperfusion Injury in Male Rats. Int J Med Sci. 2017; 14: 1163-72.

24. Depre C, Wang L, Sui X, Qiu H, Hong C, Hedhli N, Ginion A, Shah A, Pelat M, Bertrand L, Wagner T, Gaussin V, Vatner SF. H11 kinase prevents myocardial infarction by preemptive preconditioning of the heart. Circ Res. 2006; 98: 280-8

25. Small BA, Lu Y, Hsu AK, Gross GJ, Gross ER. Morphine reduces myocardial infarct size via heat shock protein 90 in rodents. Biomed Res Int. 2015; 2015: 129612

26. Csont T, Bereczki E, Bencsik P, Fodor G, Görbe A, Zvara A, Csonka C, Puskás LG, Sántha M, Ferdinandy P. Hypercholesterolemia increases myocardial oxidative and nitrosative stress thereby leading to cardiac dysfunction in apoB-100 transgenic mice. Cardiovasc Res. 2007; 76: 100-9.

27. Wang H, Joseph JA. Quantifying cellular oxidative stress by dichlorofluorescein assay using microplate reader. Free Radic Biol Med. 1999; 27(5-6): 612-6.

28. Xiao D, Dasgupta C, Chen M, Zhang K, Buchholz J, Xu Z, Zhang L. Inhibition of DNA methylation reverses norepinephrine-induced cardiac hypertrophy in rats. Cardiovasc Res. 2014; 101: 373-82.

29. Glantz SA, Parmley WW. Passive smoking and heart disease: epidemiology, physiology, and biochemistry. Circulation. 1991; 83: 1-12.

30. Lawrence J, Chen M, Xiong F, Xiao D, Zhang H, Buchholz JN, Zhang L. Foetal nicotine exposure causes $\mathrm{PKC} \varepsilon$ gene repression by promoter methylation in rat hearts. Cardiovasc Res. 2011; 89: 89-97.

31. Sancilio S, Gallorini M, Cataldi A, Sancillo L, Rana RA, di Giacomo V. Modifications in Human Oral Fibroblast Ultrastructure, Collagen Production, and Lysosomal Compartment in Response to Electronic Cigarette Fluids. J Periodontol. 2017; 88: 673-80.

32. Pelissier-Rota MA, Pelosi L, Meresse $\mathrm{P}$, Jacquier-Sarlin MR. Nicotine-induced cellular stresses and autophagy in human cancer colon cells: A supportive effect on cell homeostasis via up-regulation of Cox-2 and PGE(2) production. Int J Biochem Cell Biol. 2015; 65: 239-6.

33. Hill JA, Cao DJ, Gillette TG. Cardiomyocyte autophagy: Remodeling, repairing, and reconstructing the heart. Curr Hypertens Rep. 2009; 11: 406-11.

34. Shintani-ishida K, Saka K, Yamaguchi K, Hayashida M, Nagai H, Takemura G, Yoshida K. MDMA induces cardiac contractile dysfunction through autophagy upregulation and lysosome destabilization in rats. Biochim Biophys Acta. 2014; 1842: 691-700.

35. Valentim L, Laurence KM, Townsend PA, Carroll CJ, Soond S, Scarabelli TM, Knight RA, Latchman DS, Stephanou A. Urocortin inhibits Beclin1-mediated autophagic cell death in cardiac myocytes exposed to ischaemia/reperfusion injury. J Mol Cell Cardiol. 2006; 40: 846-52.

36. $\mathrm{Yu}$ X, Long YC, Shen HM. Differential regulatory functions of three classes of phosphatidylinositol and phosphoinositide 3-kinases in autophagy. Autophagy. 2015; 11: 1711-28.

37. Wang Y, Qin ZH. Coordination of autophagy with other cellular activities. Acta Pharmacol Sin. 2013; 34: 585-94.

38. Li D, Hu J, Wang T, Zhang X, Liu L, Wang H, Wu Y, Xu D, Wen F. Silymarin attenuates cigarette smoke extract-induced inflammation via simultaneous inhibition of autophagy and ERK/p38 MAPK pathway in human bronchial epithelial cells. Sci Rep. 2016; 6: 37751

39. Bjørkøy G, Lamark T, Johansen T. p62/SQSTM1: a missing link between protein aggregates and the autophagy machinery. Autophagy. 2006; 2: 138-9.

40. Pankiv S, Clausen TH, Lamark T, Brech A, Bruun JA, Outzen H, Øvervatn A, Bjørkøy G, Johansen T. p62/SQSTM1 binds directly to Atg8/LC3 to facilitate degradation of ubiquitinated protein aggregates by autophagy. J Biol Chem. 2007; 282: 24131-45.

41. Monick MM, Powers LS, Walters K, Lovan N, Zhang M, Gerke A, Hansdottir S, Hunninghake GW. Identification of an autophagy defect in smokers' alveolar macrophages. J Immunol. 2010; 185: 5425-35.

42. Van Jaarsveld H, Kuyl JM, Alberts DW. Exposure of rats to low concentration of cigarette smoke increases myocardial sensitivity to ischaemia/reperfusion. Basic Res Cardiol. 1992; 87: 393-9.

43. Xiao D, Wang L, Huang X, Li Y, Dasgupta C, Zhang L. Protective effect of antenatal antioxidant on nicotine-induced heart ischemia-sensitive phenotype in rat offspring. PLoS One. 2016; 11: e0150557.

44. Kirkland RA, Adibhatla RM, Hatcher JF, Franklin JL. Loss of cardiolipin and mitochondria during programmed neuronal death: evidence of a role for lipid peroxidation and autophagy. Neuroscience. 2002; 115: 587-602.

45. Scherz-Shouval R, Shvets E, Fass E, Shorer H, Gil L, Elazar Z. Reactive oxygen species are essential for autophagy and specifically regulate the activity of Atg4. EMBO J. 2007; 26: 1749-60.

46. Mathew R, Karp CM, Beaudoin B, Vuong N, Chen G, Chen HY Bray K, Reddy A, Bhanot G, Gelinas C, Dipaola RS, Karantza-Wadsworth V, White E. Autophagy suppresses tumorigenesis through elimination of p62. Cell. 2009; 137: 1062-75.

47. Chen Y, Azad MB, Gibson SB. Superoxide is the major reactive oxygen species regulating autophagy. Cell Death Differ. 2009; 16: 1040-52.

48. Hardt SE. Glycogen synthase kinase-3beta: a novel regulator of cardiac hypertrophy and development. Circ Res. 2002; 90: 1055-63.

49. Kerkela R, Kockeritz L, MacAulay K, Zhou J, Doble BW, Beahm C, Greytak S, Woulfe K, Trivedi CM, Woodgett JR, Epstein JA, Force T, Huggins GS. Deletion of GSK-3 $\beta$ in mice leads to hypertrophic cardiomyopathy secondary to cardiomyoblast hyperproliferation. J Clin Invest. 2008; 118: 3609-18. 
50. Zhai P, Sciarretta S, Galeotti J, Volpe M, Sadoshima J. Differential roles of GSK-3 $\beta$ during myocardial ischemia and ischemia/reperfusion. Circ Res. 2011; 109: 502-11.

51. Antos CL, McKinsey TA, Dreitz M, Hollingsworth LM, Zhang CL, Schreiber K, Rindt H, Gorczynski RJ, Olson EN. Dose-dependent

blockade to cardiomyocyte hypertrophy by histone deacetylase inhibitors. J Biol Chem. 2003; 278: 28930-7.

52. Stambolic V, Woodgett JR.Mitogen inactivation of glycogen synthase kinase-3 beta in intact cells via serine 9 phosphorylation. Biochem J. 1994; 303: 701-4.

53. Wu YT, Tan HL, Shui G, Bauvy C, Haung Q, Wenk MR, Ong CN, Codogno P, Shen HM. Dual role of 3-methyladenine in modulation of autophagy via different temporal patterns of inhibition on class I and III phosphoinositide 3-kinase. J Biol Chem. 2010; 285: 10850-61.

54. Ito S, Koshikawa N, Mochizuki S, Takenaga K. 3-Methyladenine suppresses cell migration and invasion of HT1080 fibrosarcoma cells through inhibiting phosphoinositide 3-kinases independently of autophagy inhibition. Int J Oncol. 2007; 31: 261-268.

55. Dai S, Wang B, Li W, Wang L, Song X, Guo C, Li Y, Liu F, Zhu F, Wang Q, Wang X, Shi Y, Wang J, Zhao W, Zhang L. Systemic application of 3-methyladenine markedly inhibited atherosclerotic lesion in ApoE-/mice by modulating autophagy, foam cell formation and immune-negative molecules. Cell Death Dis. 2016; 7: e2498. 